\title{
A MULTI-BAND INTEGRATED VIRTUAL CALIBRATION-INVERSION METHOD FOR OPEN PATH FTIR SPECTROMETRY
}

\author{
Sławomir Cięszczyk \\ Lublin University of Technology, Institute of Electronics and Information Technology, Nadbystrzycka 38A, 20-618 Lublin, Poland \\ (s.cieszczyk@pollub.pl, +48 815384311)
}

\begin{abstract}
This paper addresses problems arising from in situ measurement of gas content and temperature. Such measurements can be considered indirect. Transmittance or natural radiation of a gas is measured directly. The latter method (spectral radiation measurement) is often called spectral remote sensing. Its primary uses are in astronomy and in the measurement of atmospheric composition. In industrial processes, in situ spectroscopic measurements in the plant are often made with an open path Fourier Transform Infrared (FTIR) spectrometer. The main difficulty in this approach is related to the calibration process, which often cannot be carried out in the manner used in the laboratory. Spectral information can be obtained from open path spectroscopic measurements using mathematical modeling, and by solving the inverse problem. Determination of gas content based on spectral measurements requires comparison of the measured and modeled spectra. This paper proposes a method for the simultaneous use of multiple lines to determine the gas content. The integrated absorptions of many spectral lines permits calculation of the average band absorption. An inverse model based on neural networks is used to determine gas content based on mid-infrared spectra at variable temperatures.
\end{abstract}

Keywords: FTIR, in situ spectroscopy, open path, process diagnostics.

(C) 2013 Polish Academy of Sciences. All rights reserved

\section{Introduction}

In many industrial processes, and in environmental monitoring, it is necessary to know the concentrations of gases. Continuous measurement of exhaust gases is legally required for some processes. Requirements for measuring systems operating in harsh environments are fulfilled by various optical systems. In situ optical measurements can be performed in an active or passive way. Radiation from an object can be transferred to a spectrometer in the passive mode, or transmitted through the object in the active mode. Absorption is measured along the chosen path in the active method. "Active" refers to the need for an additional radiation source (laser or broadband). In the passive method, radiation emitted by an object or environment, such as combustion, the hot gases in a chimney, or aircraft engine exhaust gases, are measured. The two main reasons for using optical methods are inaccessibility of the process due to distance, as in atmospheric measurements, or due to difficult conditions prevailing around the process.

Fourier Transform Infrared FTIR spectroscopy is an analytical technique applicable in process and environmental gas measurement. The spectrometer records the spectra of the individual components at mid-infrared wavelengths. The main advantage of FTIR is fast and simultaneous analysis of multiple components. In many cases, a special type of open path FTIR spectrometer (OP-FTIR) is used. These spectrometers are essentially spectral radiometers that analyze the radiation coming from the environment. The primary application 
of this type of spectrometer is in the measurement of atmospheric pollutants [1,2]. This device is less common than the typical laboratory FTIR spectrometer, which is a closed system consisting of a radiation source, a test cell, a Michelson interferometer and a detector. In classical FTIR, a mixture of gases is placed in a cuvette inside the spectrometer. The absorption characteristics of the individual components allow us to determine the concentrations. The OP-FTIR spectrometer is a spectral radiometer, which in practical applications can be used as a natural process spectrum analyser (in passive mode). Using an additional source of radiation allows the analysis of the environment between the source and the spectrometer (active measurement) using transmission. The spectra obtained using OPFTIR permit the determination of the constituents and temperature of a sample. The measured concentration is then integrated along the examined path. The main advantages of OP-FTIR spectrometers are as follows [3]:

- the possibility of making measurements in situ,

- the possibility of remote sensing,

- continuous operation,

- very simple setup,

- relatively high temporal resolution.

Quantitative analysis of gases from industrial processes and environmental samples is a complicated task. The main problem is the variability of physical and chemical conditions (mainly temperature) necessary for calibration, and for determination of the concentration of certain gases (analytes). The accuracy of a procedure for the determination of components and temperature depends mainly on the algorithm used. Measurements of environmental gases and process monitoring encounter the same problems: a high content of water vapour and carbon dioxide obscuring the spectrum of interest. If very high sensitivity is required, cavity enhanced absorption spectroscopy should be used [4].

\section{Classical, virtual and synthetic calibration in spectroscopy}

The classical approach to calibration in spectroscopy requires reference samples; that is, substances whose properties are sufficiently well known. Calibration requires the determination of the relationship between the value indicated by an instrument and the value represented by the underlying reference standard [5]. At the same time, suitable conditions such as temperature and pressure must be maintained. Uncertainty estimation of calibration model should also be performed[6].

In open-path measurements, the environment changes naturally, and temperature has the greatest impact on the spectrum. Figure 1 shows changes in the transmission spectrum of methane caused by temperature.

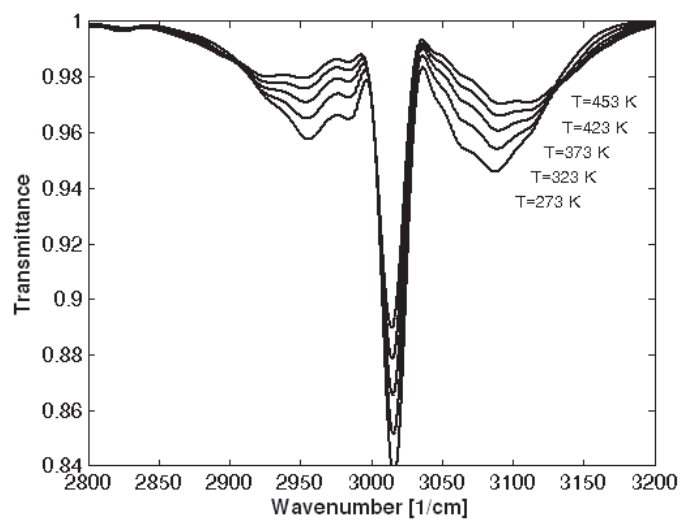

Fig. 1. Influence of temperature on the transmission spectrum of methane (Hitran simulation). 
Environmental influence must be known before the creation of the calibration model because even calibration methods as advanced as PLS (Partial Least Squares) regression, are vulnerable to non-modeled interference during calibration $[7,8,9]$. In some cases of environmental fluctuation, the LS-SVR method can be applied[10].

The building of empirical models for the calibration of open path measurements is possible only for certain types of measurements. These include measurements of atmospheric pollutants, for which similar conditions for the gas mixture can be achieved. A greater concentration of components is applied along shorter paths, and as a result, the same value of the product of path length and concentration is produced. Recently, the classical least squares method of matching spectra has been used for the analysis of air pollutants in an exhaust plume. Currently, attempts are being made to use the PLS method. This method does not require the collection of matching background spectra for measurement and calibration, which is impracticable [1]. For some types of gases, databases of FTIR spectra are created, for example, at $1.0 \mathrm{ppm}^{\cdot} \mathrm{m}, \mathrm{T}=296 \mathrm{~K}$ and atmospheric pressure [11].

Mathematical modeling is the dominant approach in the open-path measurements (Fig. 2). This results from difficulties in the reproduction of calibration conditions identical to those present in the examined object. The ability to generate an arbitrary mixture of gases under any physical-chemical conditions is the main advantage of mathematical modelling. This allows the determination of the impact (or lack thereof) of any component or parameter.

Using a high-resolution database such as Hitran [12] is the most popular method of spectral modeling. This is supported by easy adjustment of the spectrum to the instrumental line shape (ILS). ILS spectral response can be calculated theoretically, assuming the dominant effect of apodization, or experimentally, by measuring spectral lines of known shape. In the calculation of spectra using databases based on physical models of gases, all possible sources of interference can be taken into account. These databases contain coefficients for the influence of other components, temperature and pressure on the spectrum of the mixture.

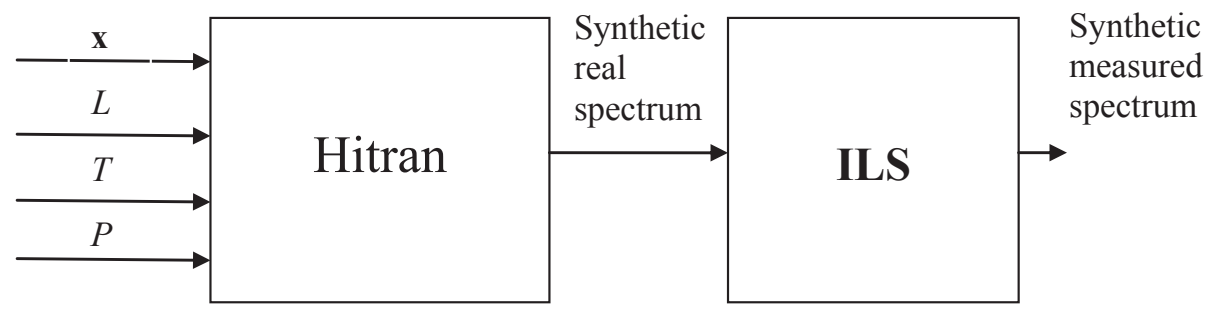

Fig. 2. Forward model for the determination of synthetic spectra, $\mathbf{x}$ - vector of individual gas content, $L$ - synthetic path length, $T$ - temperature, $P$ - pressure.

Forward modeling, which allows the calculation of synthetic spectra for specific gas compositions, can be achieved by mathematical modeling. Determination of the concentration of an unknown is based on the comparison of the measured spectrum with spectra obtained from a simple model. The most commonly encountered method for determining the content of gases uses a simple model and the nonlinear least squares method. Calibration using typical laboratory measurements therefore does not need to be performed.

Determination of gas concentrations by the inverse problem method requires good knowledge of the environment (or object) studied: the parameters can be sensitive to small changes in the data. This is a frequently encountered feature of the inverse problem of indirect measurements $[13,14]$. Comparing measured and simulated spectra is the most frequently used measure of gas concentration retrieval accuracy. The use of the additional measurement validation method is possible in a few cases. Practical OP-FTIR spectroscopy, and the 
simultaneous alternative method of gas content measurement, were performed for the gas composition 12500-360000 ppm·m CO $2\left(2150-2310 \mathrm{~cm}^{-1}\right), 55-520 \mathrm{ppm} \cdot \mathrm{m} \mathrm{CH}_{4}(2980-3090$ $\left.\mathrm{cm}^{-1}\right)$, and 20-6400 ppm $\cdot \mathrm{m} \mathrm{CO}\left(2000-2230 \mathrm{~cm}^{-1}\right.$ below $500 \mathrm{ppm} \cdot \mathrm{m}$ and $2039-2057 \mathrm{~cm}^{-1}$ for higher concentrations) $[3,15]$; in parentheses, the range of the wave number for which calculations were performed is indicated.

\section{FTIR instrumental line shape modeling}

Appropriate choice of the measurement wavelength permits inference of the concentration of a specific component, even with a substantial amount of other gases present. Unfortunately, in some cases high spectral resolution is required to distinguish rotational gas lines. In an industrial environment it is not possible to use as high a resolution as in laboratory devices. Lower resolution, however, means that the results are more susceptible to interference from other components.

An interferogram is obtained as the output of the FTIR spectrometer. To avoid large side lobes in the spectrum, the interferogram is multiplied by apodizing functions (windows). Instrumental line shape (ILS) is the Fourier transform (FT) of a particular type of apodizing function $o(z)$ :

$$
\operatorname{ILS}(v)=F T(o(z)) \text {. }
$$

For a rectangular window, this is the sinc function. For a triangular window, this is the sinc ${ }^{2}$ function (Fig. 1).

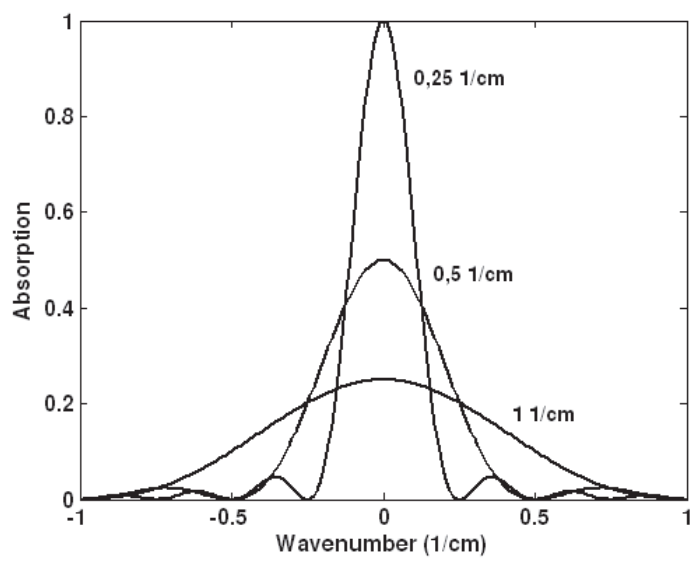

Fig. 3. Comparison of instrumental line shape for rectangular and triangular windows at 1 and $3 \mathrm{~cm}^{-1}$ resolution.

The resolution of a spectrometer is its ability to distinguish two spectral lines lying close to each other. The resolving power of an FTIR spectrometer using a very popular triangular window imposed on the interferogram (Fig. 3) depends on the maximum retardation of the interferometer's moving mirror $z_{\max }$ :

$$
\Delta v=1 / z_{\max }
$$

The spectrum obtained is the result of the convolution of the real spectrum and the ILS response of the spectrometer:

$$
H_{w}(v)=I L S(v) * H(v)=\int_{-\infty}^{+\infty} I L S\left(v^{\prime}\right) H\left(v-v^{\prime}\right) d v^{\prime},
$$


where:

$H(v)$ - real spectrum,

$\operatorname{ILS}(v)$ - Instrumental Line Shape characteristic for the measuring instrument.

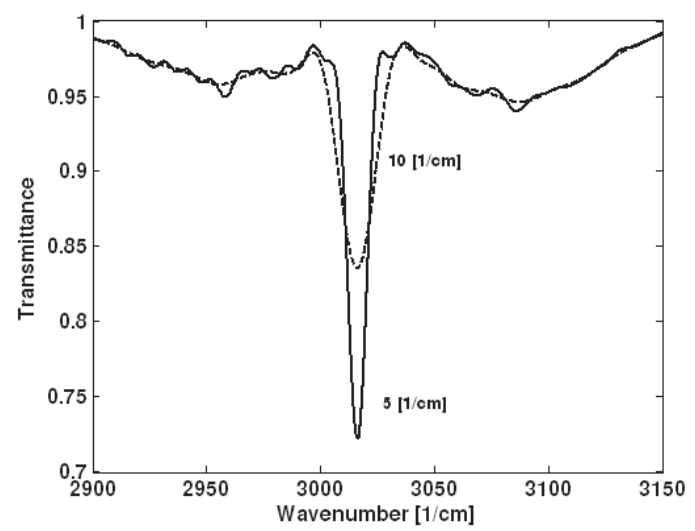

Fig. 4. Comparison of $\mathrm{CH}_{4}$ spectra (triangular apodization) at 10 and $5 \mathrm{~cm}^{-1}$ resolution.

To determine the impact of resolution on measured values, simulations of the spectra of methane at different resolutions are presented in Figures 4 and 5.

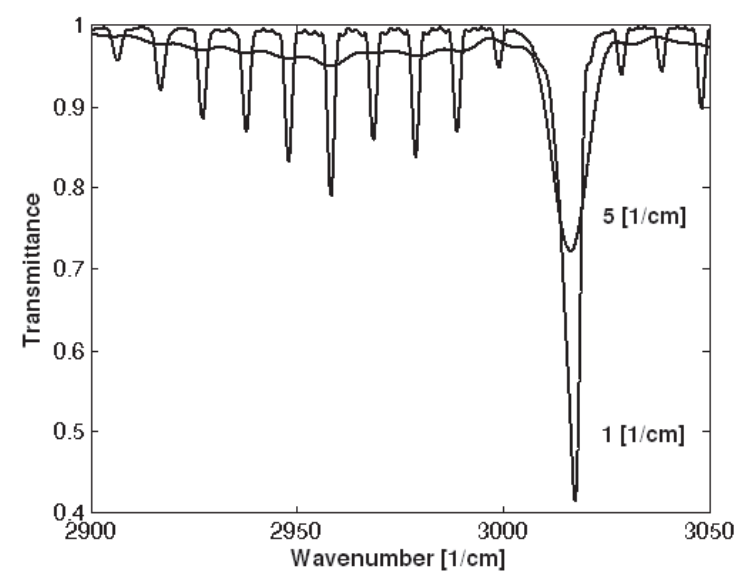

Fig. 5. Comparison of $\mathrm{CH}_{4}$ spectra (triangular apodization) at 1 and $5 \mathrm{~cm}^{-1}$ resolution.

Process exhaust gas measurements mainly employ low resolution spectroscopy. Low resolution FTIR spectrometers are simpler and more reliable than high resolution units. Low resolution measurement is faster with a greater signal-to-noise ratio. A low resolution $\left(4 \mathrm{~cm}^{-1}\right)$ was used for practical measurement of biomass pyrolysis gases in the following spectral ranges: $\mathrm{CO}_{2}\left(2400-2250 \mathrm{~cm}^{-1}\right), \mathrm{CO}\left(2240-2020 \mathrm{~cm}^{-1}\right)$ and $\mathrm{CH} 4\left(3195-1860 \mathrm{~cm}^{-1}\right)[16]$. The integrated absorption area was calculated for the ranges shown in parentheses. Due to the non-linear relationship between the area of absorption and gas content, the dependency was approximated by a second-degree polynomial. Integration of the absorption area of the bands was also used for comparison of the spectra with the spectral databases which could be used for remote measurement [17]. The integration method can reduce the number of calibration samples required. The method is resistant to perturbations (that is, robust) and more accurate 
than other methods [18]. The higher number of calibration points in typical multivariate methods results from the need to take into account every possible source of variability in the process.

\section{The method proposed for the determination of gas concentrations using open path FTIR}

To assign a value to a measured quantity, an exact relationship between the two quantities must be determined. This relationship is called a mathematical model of indirect measurements $[19,20]$. For spectroscopic measurements, such a model can be determined empirically, or by the use of mathematical modeling. Empirical models, which are created on the basis of measurements, are used for laboratory spectroscopy. For open-path measurements, such models can be produced only for selected atmospheric measurements for paths of uniform temperature. This is performed by reconstruction of paths in the laboratory, using similar concentrations and distances. For plume analysis, spectral matching using classical least squares analysis was used until recently. More recently, many tests of PLS methods have been reported. This method does not require identical background spectra for measurement and calibration, which is impractical [1].

For in situ measurements, the use of spectral modeling is very common. In the open-path measurements approach, only the direct model is known. The model parameters input are: gas mixture composition, temperature and pressure. The output of the model is a spectrum. To carry out spectral interpretation, the inverse problem has to be solved. A mathematical model of this problem exists only for simple cases, such as laboratory measurements under stable conditions, especially temperature. General mathematical inverse models do not exist in changeable conditions, or if there is a nonlinear relationship between the absorption and concentration. Thus, iterative methods using a direct model are used to solve this problem. Decreasing the difference between the measured and modeled spectra is the purpose of each iteration.

These two approaches to solve for the variables are termed the forward-model approach and inverse-model approach [21]. A forward model that transforms the gas concentrations into a spectrum can be built for open-path measurements. Thanks to this model, the concentration of a component can be determined iteratively by comparing the model output to the measured spectrum. For measurements requiring on-line results, the search for a solution to the inversemodel-based reconstruction becomes more natural. The long-term iterative process can often be avoided here. Neural networks may be the solution in such cases. They are known for their capacity for universal approximation.

The virtual calibration procedure presented above can be used for active measurements, and also for the case of a homogeneous layer at a known temperature. If the temperature of a layer changes, the more sophisticated inverse problem method is needed. The use of calibration methods which are robust to temperature change is also necessary. When building a forward model, the problem arises due to lack of knowledge for the ILS spectrometer. The ILS must be consistent with the actual parameters of the spectrometer used. Integral absorption [22] is a method which simplifies the analysis of complex spectra where ILS is not known exactly.

Collection of multi-line spectra gives lower limits of detection in comparison with singleline measurement. The limit of detection for multi-line fitting is dependent on the shape of the spectra. However, it decreases with an increase in the integrated area of absorption [23]. Multi-line fitting models allow the utilisation of spectral sections with weak absorption. These spectral sections are then placed in an area where the sources and detector manufacturing are strongly developed and cheap [24]. The multi-line integrated absorption spectroscopy method 
is also used to analyze data collected using lasers. This method relies on the integrated absorption parameter of many closely spaced lines, which gives a 15 -fold improvement in the detection limit compared to the standard method [25]. The method of integrated absorption in several bands can eliminate the need for an accurate determination of the spectrometer ILS, and of errors resulting from the inaccurate calibration of a wavelength.

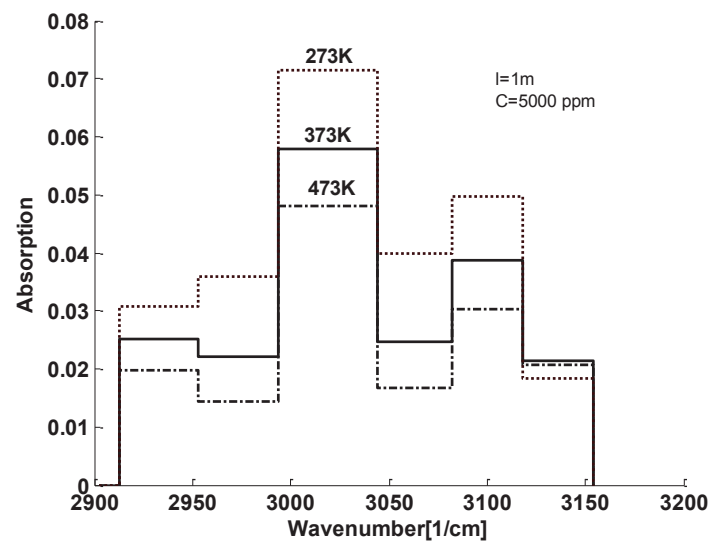

Fig. 6. $\mathrm{CH}_{4}$ Methane spectra after the integration of 6 bands for 3 different temperatures.

For methane, different parts of the spectrum can be selected in the range $2800-3200 \mathrm{~cm}^{-1}$. The spectrum depicted in Fig. 6 was divided into 6 bands. For each band the integrated absorption was calculated. Figure 6 shows the influence of temperature on the absorption value in each band.

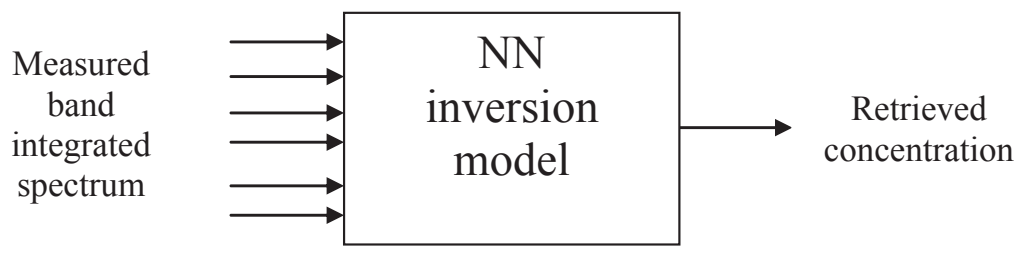

Fig. 7. The inverse model based on the neural network used in the determination of concentration on the basis of spectra integrated in 6 bands.

All six integrated bands permit the determination of the concentration of methane. The synthetic calibration set can be obtained by calculation for a specified number of concentrations. Following that, it can be used to determine a synthetic calibration model. A neural network (Fig. 7) is used as an inverse model because of nonlinear dependence of the spectrum on temperature and concentration. Such a model may be utilized for the determination of methane concentration on the basis of variable- temperature spectra. Figure 8 shows neural network properties as an inverse model for synthetic data. Over most of the concentration range, the absolute error is limited to $2 \mathrm{ppm} \cdot \mathrm{m}$ (Fig. 9), increasing only above $900 \mathrm{ppm} \cdot \mathrm{m}$. A multi-layer perception-based neural network was used for model construction. By use of the direct problem, a teaching and validation network set was prepared. 


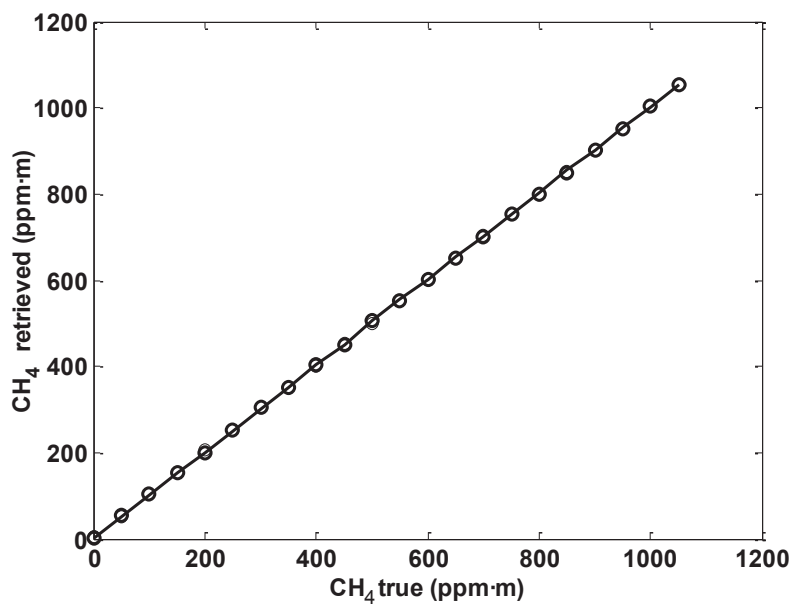

Fig. 8. The relationship between the value derived from the inverse model and the real $\mathrm{CH}_{4}$ concentration from the simulation.

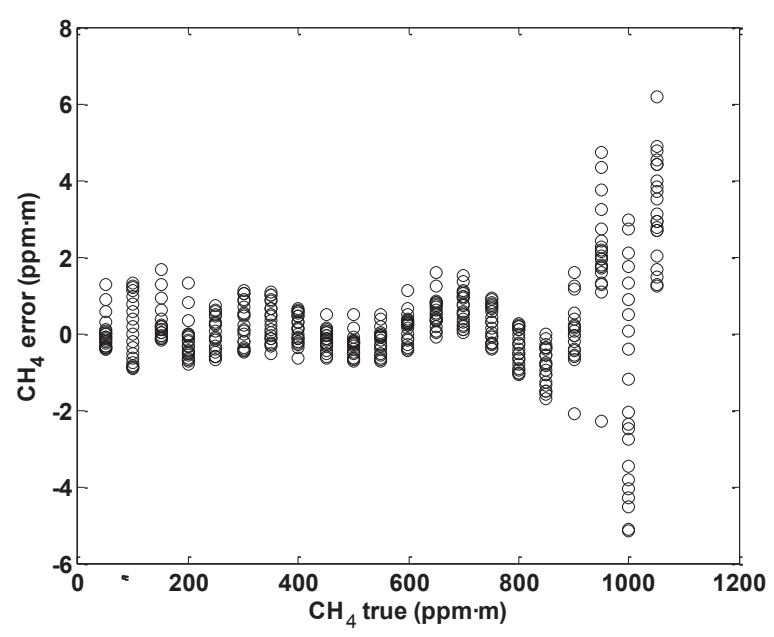

Fig. 9. The absolute error for the simulated recovery of methane.

Empirical confirmation of the depicted method was carried out using two types of data. The first dataset was $\mathrm{CH}_{4}$ measurement over a length of $2.25 \mathrm{~m}$, concentration $400.5 \mathrm{ppm}$, resolution $0.25 \mathrm{~cm}^{-1}$ and temperature $100^{\circ} \mathrm{C}[26]$; therefore, the integrated absorption over the path was $901.124 \mathrm{ppm} \cdot \mathrm{m}$. The neural network inverse model result was $967 \mathrm{ppm} \cdot \mathrm{m}$, a relative error of $7.3 \%$. The second measurement was carried out by the author. The path length was 8 $\mathrm{m}$, and hot burner elements were used as the radiation source. Gases generated by biomass gasification flowed through the chamber [27]. The estimated $\mathrm{CH}_{4}$ concentration was 1070 $\mathrm{ppm} \cdot \mathrm{m}$. 


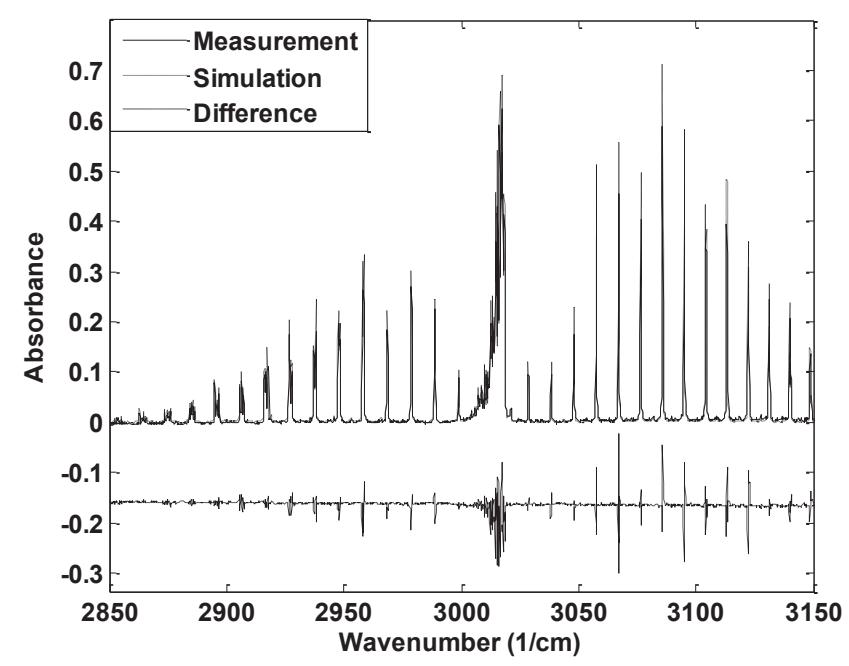

Fig. 10. Comparison of the measured spectrum (EPA) and that obtained from the inverse model $\left(0.25 \mathrm{~cm}^{-1}\right.$ resolution).

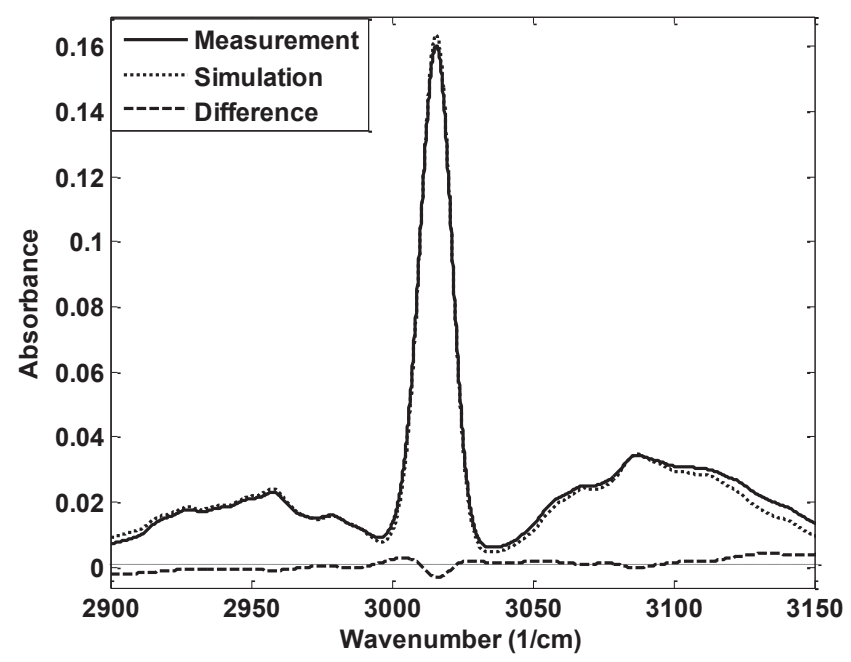

Fig. 11. Comparison of the measured spectrum (EPA) and that obtained from the inverse model $\left(5 \mathrm{~cm}^{-1}\right.$ resolution).

In the high-resolution spectrum (Fig. 10), there are differences between measured and simulated spectra, caused by inaccuracy in the modeled ILS. After reducing the resolution by convolution of both spectra with the Gaussian function $\left(5 \mathrm{~cm}^{-1}\right.$ width), the ILS inaccuracy problem was eliminated, and the differences between model and measurement were small, as shown in Fig. 11. 


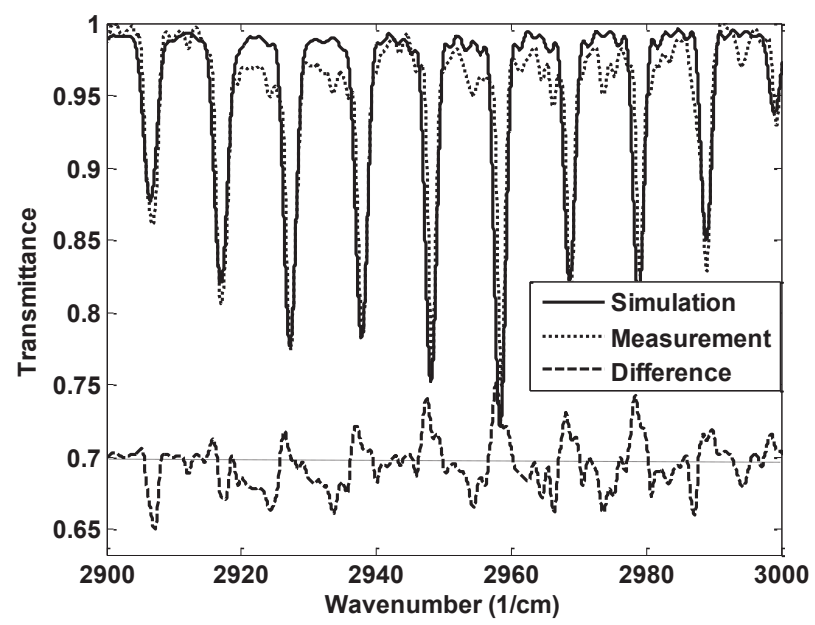

Fig. 12. Comparison of the measured spectrum (biomass gasification) and that obtained from the inverse model $\left(1 \mathrm{~cm}^{-1}\right.$ resolution).

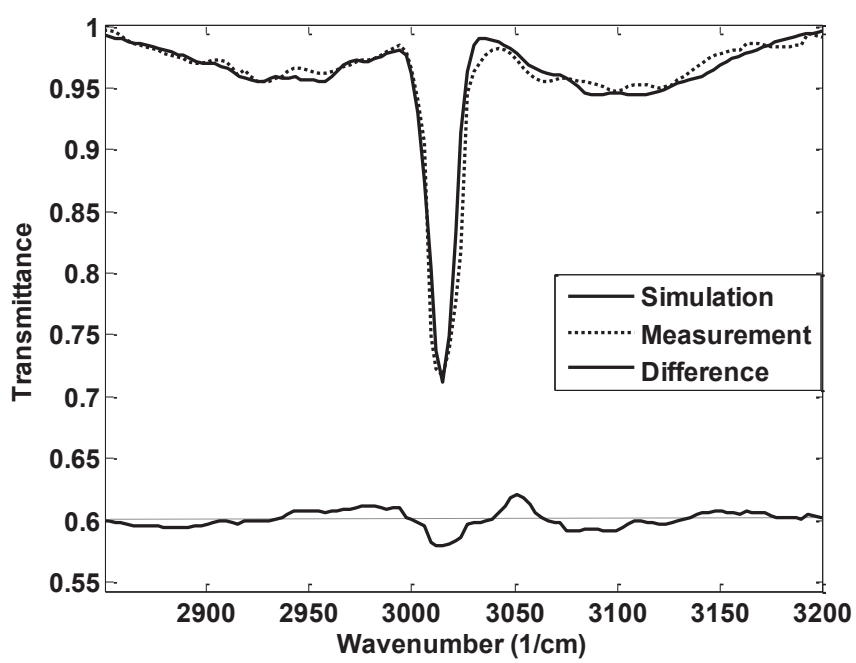

Fig. 13. Comparison of the measured spectrum (biomass gasification) and that obtained from the inverse model $\left(5 \mathrm{~cm}^{-1}\right.$ resolution).

For biomass gasification measurements, the radiation source exhibited larger fluctuations, creating obstacles to proper modeling of the background spectrum. This can be seen from the larger differences between the measured and simulated spectra that occur between rotational lines (Fig. 12). The low resolution spectrum is a better fit to the simulation (Fig. 13).

\section{Summary}

This paper presents a method to determine gas concentrations using in situ measurements. A typical analytical laboratory calibration procedure is presented, for which maintaining consistent physical and chemical conditions are critical. For in situ use in the abovementioned atmospheric or process measurements, such as combustion or biomass processing, 
these requirements are difficult to sustain. Therefore, standard analytical calibration cannot be used. For this reason, mathematical modeling methods are used for the generation of so-called virtual or synthetic spectra. For testing a homogeneous gas layer, simulated spectra may be used for virtual calibration. The cuvette, with a mixture of gases and appropriate physicalchemical conditions, is replaced by mathematical modeling. Using data from simulations and well-known chemometric methods, a calibration model is created. Using this model, gas concentrations are determined from the measured spectra. Due to difficulties in accurate ILS modeling, spectral line integration can be used to improve the accuracy of concentration determinations. Bands are formed by integrating several lines. Several bands can be used simultaneously to determine the concentration of the desired component. Where the relationship between absorbance and concentration is non-linear, neural networks may be utilized as the inverse model. Nonlinearity occurs where the product of concentration and path-length is large. As an example of the method, the mid-infrared spectrum of methane is presented. A model prepared from synthetic data demonstrated itself against both synthetic evaluation data and two types of measured data.

\section{References}

[1] Griffiths P.R., Shao L., Leytem A. B. (2009): Completely automated open-path FTIR spectrometry, Anal Bioanal Chem, 393, 45-50.

[2] Kastek M., Piątkowski T., Trzaskawka P. (2011): Infrared imaging Fourier transform spectrometer as the stand-off gas detection system, Metrology and Measurement Systems, 18(4), 607-620,

[3] Smith T.E.L., Woodster M.J,, Tattaris M., Griffith D.W.T.(2010): Absolute accuracy and sensitivity analysis of OP-FTIR retrievals of $\mathrm{CO} 2, \mathrm{CH} 4$ and $\mathrm{CO}$ over concentrations representative of clean air and polluted plumes, Atmospheric Measurement Techniques, 4, 97-116.

[4] Wojtas J., Czyżewski A., Stacewicz T., Bielecki Z.(2006): Sensitive detection of NO2 with cavity enhanced spectroscopy, Optica Applicata 36(4), 461-465.

[5] Cuadros-Rodrigues L., Gamiz-Gracia L., Almansa-Lopez E.(2001): Calibration in chemical measurement process: I. A metrological approach, Trends in analytical chemistry, 20(4), 195-206.

[6] Lorber A., Kowalski B.R.(1988): Estimation of prediction terror for multivariate calibration, Journal of Chemometrics, 2(2), 93-109.

[7] Haaland D.M.(2000): Synthetic multivariate models to accommodate unmodeled interfering spectral components during quantitative spectral analyses, Applied Spectroscopy, 54(2), 246-254.

[8] Shao L., Roske C.W., Griffiths P.R.(2010): Detection of chemical agents in the atmosphere by open-path FTIR spectroscopy under condition of background interference: II. Fog and rain, Anal Bioanal Chem, 397, 1521-1528.

[9] Shao L., Roske C.W., Griffiths P.R.(2010): Detection of chemical agents in the atmosphere by open-path FTIR spectroscopy under condition of background interference: I. High-frequency flashes, Anal Bioanal Chem, 397, 1511-1513.

[10] Barman I., Dingari N.C., Singh G.P., Soares J.S., Dasari R.R., Smulko J.M.(2012): Investigation of NoiseInduced Instabilities in Quantitative Biological Spectroscopy and Its Implications for Noninvasive Glucose Monitoring, Analytical Chemistry, 84(19), 8149-8156.

[11] Johnson T.J. at all(2010): An infrared spectral database for detection of gases emitted by biomass burning, Vibrational Spectroscopy, 53, 97-102.

[12] Rothman L. S., at all(2005): The HITRAN 2004 molecular spectroscopic database, Journal of Quantitative Spectroscopy and Radiative Transfer, 96, 139-204.

[13] Szczuczyński D.K., Mroczka J.(2009): Comparing the quality of solutions of inverse problem in nephelometric and turbidimetric measurements, Optica Applicata 39(3), 521-531.

[14] Mroczka, J., Szczuczyński, D. (2012). Simulation research on improved regularized solution of inverse problem in spectral extinction measurements, Applied Optics, 51(11), 1715-1723. 
[15] Smith T.E.L., Woodster M.J., Tattaris M(2010).: Open-Path FTIR spectroscopy of CO2, CH4 \& CO: Experimental accuracy evaluation for ambient to highly polluted concentrations, Proceedings of the Remote Sensing and Photogrammetry Society Conference, Remote Sensing and the Carbon Cycle, Burlington House, London, 1-4.

[16] Granada E., Equia P., Vilan J.A., Comesana J.A., Comesana R.(2012): FTIR quantitative analysis technique for gases. Application in a biomass thermochemical process, Renewable Energy 41, 416-421.

[17] Sharpe S.W., Johnson T.J., Sams R.L., Chu P.M., Rhoderick G.C., Johnson P.A.(2004): Gas-Phase Databases for Quantitative Infrared Spectroscopy, Applied Spectroscopy, 58(12), 1452-1461.

[18] Brulls M., Folestad S., Sparen A., Rasmuson A., Salomonsson J.(2007): Applying spectral peak area analysis in near-infrared spectroscopy moisture assays, Journal of Pharmaceutical and Biomedical Analysis, 44, 127-136.

[19] Mroczka J., Szczuczyński D.(2009): Inverse problems formulated in terms of first-kind Fredholm integral equations in indirect measurements, Metrology and Measurement Systems, 16(3), 333-357.

[20] Mroczka J., Szczuczyński D.(2010): Improved regularized solution of the inverse problem in turbidimetric measurements, Applied Optics, 49(24), 4591-4603.

[21] Morawski R.Z.(2006): Spectrophotometric applications of digital signal processing, Measurement Science and Technology, 17, R117-R144.

[22] Sepman A.V., Den Blanken R., Scheppers R., De Goey L.P.H.(2009): Quantitative Fourier Transform Infrared Diagnostics of the Gas-Phase Composition Using the Hitran Database and the Equivalent Width of the Spectral Features, Applied Spectroscopy, 63(11), 1211-1222.

[23] Adler F., Maslowski P., Foltynowicz A., Cossel K., Briles T., Hartl I., Ye J.: Mid-infrared Fourier transform spectroscopy with a broadband frequency comb, Optics Express, 18(21), 21861-21872.

[24] Cubillas A.M., Lazaro J.M., Conde O.M., Petrovich M.N., Lopez-Higuera J.M.(2009): Multi-Line Fit Model for the Detection of Methane at v2+2v3 Band using Hollow-Core Photocic Bandgap Fibres, Sensors, 9, 490-502.

[25] Karpf A., Rao G.N.(2009): Enhanced sensitivity for the detection of trace gases using multiple line integrated absorption spectroscopy, Applied Optics, 48(27), 5061-5066.

[26] U. S. Environmental Protection Agency: Additional Fourier Transform Infrared (FTIR) Spectra (January 2013) http://www.epa.gov/ttn/emc/ftir/addcas.html.

[27] Wójcik W., Cięszczyk S., Golec T.(2010): Narrow-band spectra models for diagnostic of gases produced during the biomass production, Environmental Engineering III (ed. L. Pawłowski, M. Dudzińska, A. Pawłowski), CRC Press, 597-601. 